\title{
Effect of Friction Deformation on the Structure and Properties of a Metastable Austenitic Chromium-Nickel Steel
}

\author{
V. R. Baraz* and O. N. Fedorenko \\ B.N. Yeltsin Ural Federal University, ul. Mira 28, Yekaterinburg, 620002 Russia \\ *e-mail:vrbaraz@mail.ru \\ Received July 20, 2011
}

\begin{abstract}
The effect of surface friction deformation on the phase composition, structure, and strength properties of a ribbon produced from a chromium-nickel steel with metastable austenite is studied. It is shown that friction processing intensifies the $\gamma-\alpha$ transformation, creating favorable conditions for the formation of a highly dispersed structure in a thin surface layer and, thus, increasing the microhardness, the elastic limit, the fatigue stability, and the Bauschinger effect.
\end{abstract}

DOI: $10.1134 / \mathrm{S} 0036029512100047$

\section{INTRODUCTION}

There are various methods of surface strengthening mechanical processing of steel parts based on the translational motion of tool over the surface of a material [1]. These methods include surface friction deformation, namely, the processing of an immobile workpiece by an indenter moving under a load. A similar scheme of loading in the surface layer of a material can induce a bulk state of stress with a high specific pressure due to the action of compressive and shear loads. As a result, plastic deformation can proceed by not only shear but also rotational mechanisms. Similar processing allows the formation of a nanocrystalline structure in a thin surface layer [2,3].

Friction processing seems to be quite efficient for parts with a small cross section, since a thin hardened surface layer is comparable with the part thickness. In this work, we study the effect of friction loading under sliding friction conditions on the formation of the phase composition, fine structure, and strain hardening of a ribbon made of a chromium-nickel austenitic steel with the metastable $\gamma$ phase. We assumed that the deformation-induced $\gamma-\alpha$ polymorphic transformation during additional surface friction processing creates conditions for the formation of higher functional properties.

\section{EXPERIMENTAL}

We studed steel belonging to austenitic steels wiith the metastable $\gamma$-phase; the contents of the main elements in the steel are as follows (wt \%): $0.12 \mathrm{C}$, 16.6 Cr, 8.0 Ni, 1.39 Si, 1.93 Mn, 1.18 Mo, and 0.8 V.

A wire workpiece $2.16 \mathrm{~mm}$ in diameter was quenched from $1070^{\circ} \mathrm{C}$ to form a supersaturated $\gamma$ solid solution, deformed by drawing (reduction) by $40 \%$, and flattened into a $0.42 \times 3.55-\mathrm{mm}$ ribbon. At the final stage of flattening, the ribbon was subjected to strain aging at $470^{\circ} \mathrm{C}$ for $1 \mathrm{~h}$. Additional friction processing was performed before aging.

Friction loading was performed on a testing machine with a cylindrical indenter having the working diameter of $6 \mathrm{~mm}$ and made from high-strength alloy. To prevent the sticking of the indenter to a specimen, friction was carried out using an MC-20 lubricant. Loading was performed at a pressure of $200 \mathrm{~N}$ and a sliding speed of $0.13 \mathrm{~m} / \mathrm{s}$. Such conditions almost excluded heating of the material in processing. The indenter diameter significantly overlapped the ribbon in width $(3.5 \mathrm{~mm})$; because of this, the indenter was moved in a reverse manner with no transverse displacement. The indenter stroke was $75 \mathrm{~mm}$, and the number of back and forth strokes was 200. A specimen was processed from either side.

X-ray diffraction (XRD) study was carried out on a DRON-2 diffractometer ( $\mathrm{Co} K_{\alpha}$ radiation) to determine phase composition and also to estimate texture by determining pole density $P_{h k l}$ of (111), (200), (220), and (311) reflections [4].

Electron-microscopic examination of thin foils prepared from the flattened ribbon was performed using an EMV-100L microscope.

The microhardness of ribbon specimens was measured on a PMT- 3 device at a load of $0.5 \mathrm{~N}$. The elastic limit was found using pure bending with a residual strain tolerance of $0.03 \%$ [5]. The fatigue tests of cantilever ribbon specimens were carried out at a working stress of $700 \mathrm{MPa}$. The vibration amplitude was $13 \mathrm{~mm}$, the loading frequency was 1300 cycles/min, and the number of repeated measurements was $6-8$. 
Table 1. Phase composition of the austenitic steel subjected to various types of processing

\begin{tabular}{l|c|c}
\hline \multicolumn{1}{c|}{ Processing } & $\gamma$ phase, $\%$ & $\alpha$ phase, $\%$ \\
\hline Quenching & 100 & 0 \\
Quenching + deformation $40 \%$ & 80 & 20 \\
Quenching + deformation $40 \%+$ aging $470^{\circ} \mathrm{C}, 1 \mathrm{~h}$ & 84 & 16 \\
Quenching + deformation $40 \%+$ friction & 68 & 32 \\
Quenching + deformation $40 \%+$ friction + aging $470^{\circ} \mathrm{C}, 1 \mathrm{~h}$ & 70 & 30 \\
\hline
\end{tabular}

The Bauschinger effect (so-called Bauschinger strain) was found from the ratio of the difference between the elastic limits of forward $\left(\sigma_{0.03}^{f}\right)$ and reverse $\left(\sigma_{0.03}^{r}\right)$ loading to the elastic limit of forward loading [6], $B=100\left(\sigma_{0.03}^{f}-\sigma_{0.03}^{r}\right) / \sigma_{0.03}^{f}$.

\section{RESULTS AND DISCUSSION}

According to XRD data, the steel demonstrates a single-phase austenitic state after quenching (Table 1). Cold deformation (drawing and subsequent flattening) leads to active development of the polymorphic $\gamma-\alpha$ transformation and the formation of a marked amount of martensite. In this case, additional friction processing increases the content of strain-induced martensite by a factor of 1.5-2, and its total fraction can exceed $30 \%$. In addition, strain aging at $470^{\circ} \mathrm{C}$ slightly decreases the $\alpha$-phase content due to the initial stages of the reverse martensite transformation.

The fine structure of the steel in the deformed state is shown in Figs. 1 and 2. Processing without friction results in the typical structure of a deformed austenite matrix characterized by a high dislocation density and the presence of deformation twins and $\alpha$-phase martensite (Fig. 1).

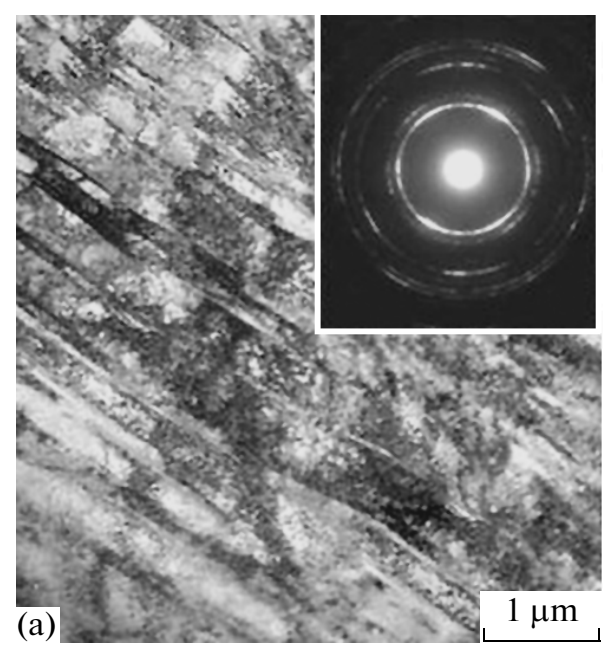

Additional deformation by sliding friction markedly changes the character of the fine structure in the friction contact zone. It is expressed in substantial fragmentation of the structural elements, which is accompanied by a tendency to their crystallographic misorientation (Fig. 2). A dark-field image indicates a relatively high fraction of the martensitic $\alpha$ phase. The thickness of the strain-hardened layer formed as a result of friction loading is $10 \mu \mathrm{m}$. The peculiarity of the diffraction pattern should be noted as follows: numerous point reflections are observed instead of typical diffuse Debye rings. In appearance, this diffraction pattern is characteristic of primary recrystallization in the material, although the conservation of a hardened state in this case is confirmed by the high strength properties. Actually, additional friction processing led to an increase in the elastic limit from 1220 to $1390 \mathrm{MPa}$ and in the microhardness from 2450 to $3390 \mathrm{HV}$ (after the complete cycle of thermomechnical hardening by the scheme quenching-strain hardening-aging).

This result can be due to the formation of a complex state of stress induced by friction loading. Under high-pressure sliding friction conditions, rotational deformation can also be developed along with shear deformation occurring via slip and twinning. This

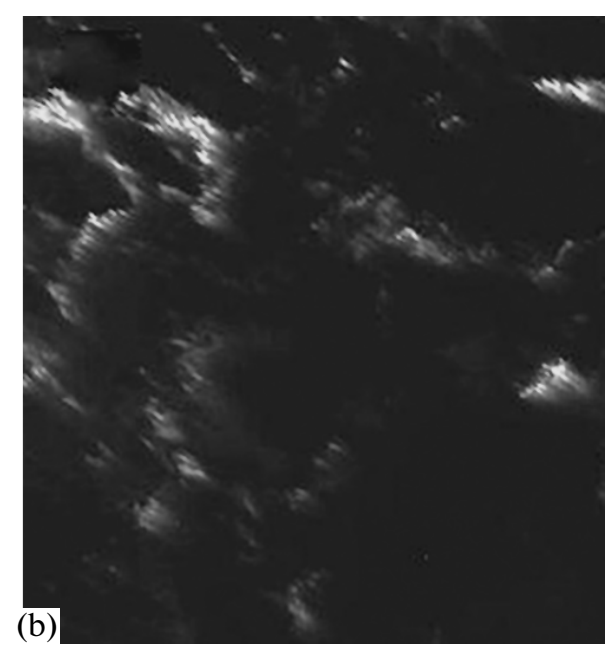

Fig. 1. Structure of a deformed ribbon specimen of the austenitic steel after hardening with no friction $($ TEM, $\times 20000)$ : (a) brightfield image and electron diffraction pattern and (b) dark-field image taken with the (011) $\alpha$ reflection. 

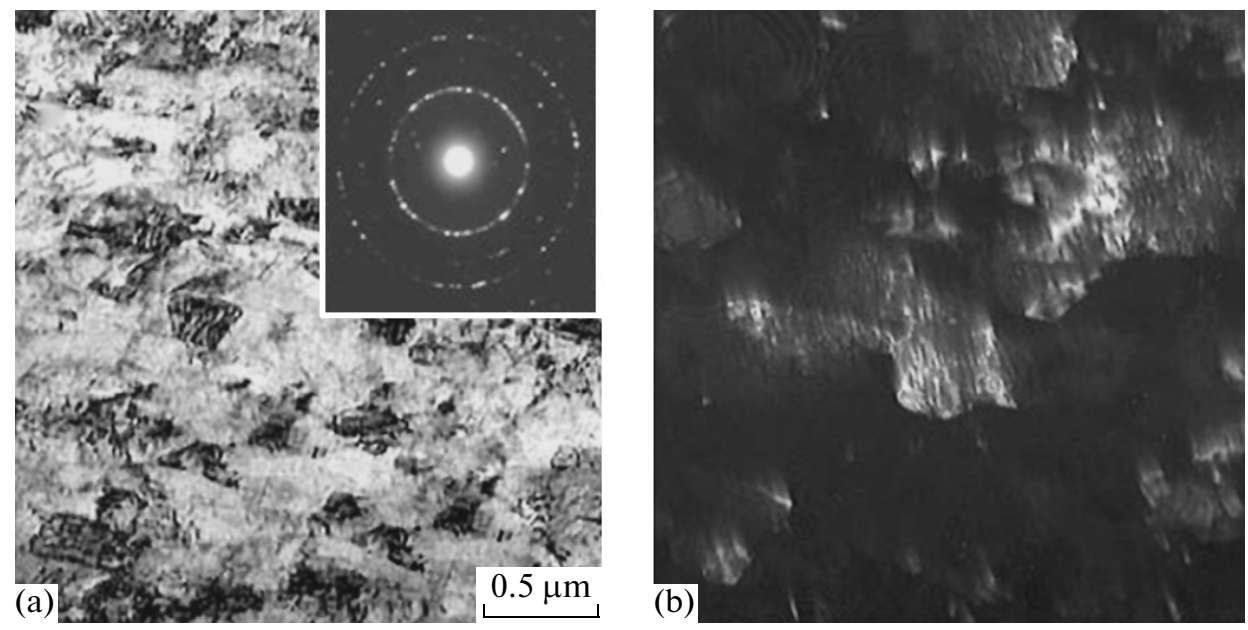

Fig. 2. Structure of a deformed ribbon specimen of the austenitic steel after hardening with friction (TEM, $\times 30000)$ : (a) brightfield image and electron diffraction pattern and (b) dark-field image taken with the (011) $\alpha$ reflection.

behavior is assumed to result in significant plastic deformation accompanied by not only anomalously high grain fragmentation but also their crystallographic rotation at a high-angle misorientation.

We can note the following expected results: a significant influence of additional friction processing of the ribbon on the properties measured during bending loading. This influence is most substantial during cyclic tests. Cold deformation with subsequent hardening friction processing markedly increases the fatigue fracture resistance. The strength characteristics $\left(H V, \sigma_{0.03}\right)$ increase by $15-30 \%$, and the fatigue properties are more sensible to the friction hardening: the number of cycles to failure increases by a factor of 2.5, from 4580 to 11640 .

The relative value Bauschinger strain characterizing the Bauschinger effect after the complete cycle of thermomechanical processing (quenching-strain hardening-aging) is dependent on the processing by sliding friction: $B=42 \%$ without processing and $B=$ $50 \%$ when this processing is applied. Friction deformation increases the apparent elastic limit during forward loading and decreases it during repeated deformation in the reverse direction. As a result, the Bauschinger strain increases.

These data agree well with the available data on a more marked Bauschinger effect in a more hardened material. The result can be explained from a stand- point of the existing dislocation models. For example, additional friction provides effective dislocation multiplication and, thus, increases the strain resistance during forward loading; correspondingly, the shear resistance markedly is more pronounced during reverse loading [6].

We studied the texture formation in the strained specimens and the effect of friction processing on this process. Table 2 gives the data characterizing the pole density for all reflections. A higher pole density is observed for 220 lines. This means that a $\{110\}$-type reflecting plane is predominantly oriented in the ribbon plane. It should be noted that the application of friction significantly weakens the 220 reflection, which can be considered as a decrease in the texture sharpness in the specimens.

It is known [7] that the rolling deformation of austenitic steels forms the standard $\{110\}\langle 112\rangle$ texture in them. Our study confirms the predominant arrangement of a $\{110\}$-type plane parallel to the ribbon plane. Here, it should be noted that the flattened ribbon can have a quite complex texture because of the peculiarities of flattening deformation: a wire is subjected to drawing and, then, rolling. As a result, an axial texture (texture of drawing) forms at the initial stages of such a complex deformation and then transforms into a rolling texture at the final stage (during flattening). The rolling texture dominates after deformation according to such a complex scheme.

Table 2. Pole density distribution

\begin{tabular}{|c|c|c|c|c|}
\hline Processing & $P_{111}$ & $P_{200}$ & $P_{220}$ & $P_{311}$ \\
\hline Quenching + deformation $40 \%+$ aging $470^{\circ} \mathrm{C}, 1 \mathrm{~h}$ & 0.6 & 0.2 & 4.3 & 0.7 \\
\hline Quenching + deformation $40 \%+$ friction + aging $470^{\circ} \mathrm{C}, 1 \mathrm{~h}$ & 1.0 & 0.1 & 2.1 & 0.8 \\
\hline
\end{tabular}


These results show that the rolling texture weakens upon additional deformation by sliding friction. This effect is likely due to the complex state of stress induced by friction loading, i.e., the development of rotational plasticity along with shear deformation. This development leads to very strong plastic deformation accompanied by not only anomalously high grain fragmentation but also crystallographic rotation of grains.

\section{CONCLUSIONS}

Surface friction processing of ribbon specimens of a chromium-nickel austenitic steel with the metastable $\gamma$ phase activates the deformation-induced $\gamma-\alpha$ transformation, promoting the formation of a highly dispersed structural state characterized by angular rotation of fragmented microvolumes. Similar processing by external friction increases the strength properties (apparent elastic limit, microhardness) of the ribbon by $15-30 \%$ and more substantially (by a factor of 2.5 ) influences the fatigue properties. In addition, the sharpness of the strain-induced texture weakens, and the resistance of the steel to repeated loading with the opposite sign decreases (Bauschinger effect).

\section{REFERENCES}

1. L. G. Odintsov, Hardening and Finishing of Parts by Surface Plastic Deformation: A Handbook (Mashinostroenie, Moscow, 1987).

2. L. G. Korshunov, A. V. Makarov, and I. L. Chernenko, "Nanocrystalline Friction Structures in Steels and Alloys, Their Strength and Tribological Properties," in Development of Ideas of Academician V.D. Sadovskii (Kvist, Yekaterinburg, 2008), pp. 218-241.

3. A. V. Makarov, L. G. Korshunov, V. B. Vykhodets, T. E. Kurennykh, and R. A. Savrai, "Effect of Strengthening Friction Treatment on the Chemical Composition, Structure, and Tribological Properties of a HighCarbon Steel,” Phys. Met. Metallogr. 110 (5), 507-520 (2010).

4. S. S. Gorelik, L. M. Rastorguev, and Yu. A. Skakov, $X$-Ray Diffraction and Electron-Optical Analyses (Metallurgiya, Moscow, 1990).

5. V. Ya. Zubov, "Determination of the Apparent Elastic Limit of a Spring Ribbon upon Pure Bending," Zav. Labor., No. 12, 1486-1487 (1949).

6. S. V. Grachev, Heat Treatment and the Resistance of Alloys to Repeated Loading (Metallurgiya, Moscow, 1976).

7. G. Wasserman and I. Greven, Texture of Metallic Materials (Metallurgiya, Moscow, 1969). 